\title{
Retropharyngeal Abscess Complicated with Torticollis: Case Report and Review of the Literature
}

\author{
Jun Hasegawa, ${ }^{1}$ Masaru Tateda, ${ }^{1}$ Hiroshi Hidaka, ${ }^{1}$ Shun Sagai, ${ }^{1}$ \\ Ayako Nakanome, ${ }^{1}$ Katsunori Katagiri, ${ }^{1}$ Masahiro Seki, ${ }^{2}$ Yukio Katori ${ }^{3}$ \\ and TOSHIMITSU KOBAYASHI ${ }^{3}$ \\ ${ }^{1}$ Department of Otorhinolaryngology, Iwaki Kyoritsu General Hospital, Iwaki, Japan \\ ${ }^{2}$ Department of Orthopaedics, Iwaki Kyoritsu General Hospital, Iwaki, Japan \\ ${ }^{3}$ Department of Otorhinolaryngology-Head and Neck Surgery, Tohoku University Graduate School \\ of Medicine, Sendai, Japan
}

Hasegawa, J., Tateda, M., Hidaka, H., Sagai, S., Nakanome, A., Katagiri, K., Seki, M., Katori, Y. and Kobayashi, T. Retropharyngeal Abscess Complicated with Torticollis: Case Report and Review of the Literature. Tohoku J. Exp. Med., 2007, 213 (1), 99-104 - Retropharyngeal abscess commonly develops among infants and small children, and is associated with the severe inflammation of the retropharyngeal lymph nodes located in the retropharyngeal space. Retropharyngeal abscess causes cervical pain, swelling, contracture of the neck, and in rare cases inflammatory torticollis, all of which result from an inflammatory process that irritates the cervical muscles, nerves or vertebrae. Here we report a rare case of retropharyngeal abscess with a complication of torticollis. A 4-yearold girl suffered from severe retropharyngeal abscess spreading through the deep cervical fascia, as judged by magnetic resonance imaging of the neck. Blood analysis showed high degree of inflammatory reactions, and so the patient was transferred to our hospital ward. The inflammation caused spasms of the prevertebral muscles, eventually leading to torticollis. The surgical drainage was performed immediately under general anesthesia, and an anti-inflammation therapy with intravenously administered meropenem trihydrate and clindamycin was used together with traction therapy to relieve the symptoms of the patient. We must be careful about the existence of epidural abscess and infectious spondylitis when the retropharyngeal abscess causes torticollis. In conclusion, an anti-inflammation therapy using antibiotics, along with traction therapy, was effective to relieve the symptoms. In addition to repeated clinical examinations, cooperation with orthopedists and careful follow-up are necessary. We also discussed the relationship between acute torticollis and retropharyngeal abscess. —— retropharyngeal abscess; torticollis; danger space; deep neck fascia; traction therapy

(C) 2007 Tohoku University Medical Press

Received June 11, 2007; revision accepted for publication July 27, 2007.

Correspondence: Jun Hasegawa, Department of Otorhinolaryngology, Iwaki Kyoritsu General Hospital, 16 Kuzehara, Uchigoh Mimayamachi, Iwaki 973-8555, Japan.

e-mail: junha@orl.med.tohoku.ac.jp 
Retropharyngeal abscess commonly occurs among infants and small children (Sharma et al. 1998). The severe inflammation of the retropharyngeal lymph nodes in the retropharyngeal space forms the abscess. The retropharyngeal space is divided into anterior and posterior areas by the alar part of prevertebral fascia (Fig. 1). It is generally believed that the retropharyngeal inflammation within the anterior areas will not spread directly to the prevertebral area. However, if it spreads across the prevertebral fascia and reaches the posterior areas, severe complications may develop, such as mediastinal and epidural abscess (Shumrick and Sheft 1991). Therefore, early diagnosis and treatment are indispensable to prevent complications.

Inflammatory torticollis is caused by muscle contracture or swollen lymph nodes from external injuries or cold, and sets up stiffness, rigidity, and torsion of neck (Bredenkamp and Maceri 1990; Mezue et al. 2002).

In our report, a rare case of retropharyngeal abscess with torticollis is the subject. The effectiveness of traction therapy for the torticollis after surgical drainage and anti-inflammation therapy with antibiotics is also discussed.

\section{Case Report}

\section{History of events}

The patient, a 4-year-old girl, developed fever and pharyngeal pain around March 25, 2006, and was examined in the pediatrics ward of a nearby general hospital. Blood analysis revealed high inflammatory reactions such as hyperthermia, marked pharyngeal and neck pain, and poor ingestion, and was carried to an ER for closer investigations. The previous physician had prescribed an acetaminophen suppository for her pharyngeal pain. The head was tilted sideways, toward the left chest. Temperature was between $37^{\circ} \mathrm{C}$ and $38^{\circ} \mathrm{C}$. White blood cell count (WBC) was 23,900/ul; C-reactive protein (CRP), $13.14 \mathrm{mg} / \mathrm{dl}$ and creatine kinase (CPK), $264 \mathrm{IU} / \mathrm{l}$. An intravenous antibiotic drip was therefore administered. Magnetic resonance imaging (MRI) of the neck on the same day found a mass suggesting retropharyngeal abscess (Fig. 2), and conspicuous tumefaction of the prevertebral muscles

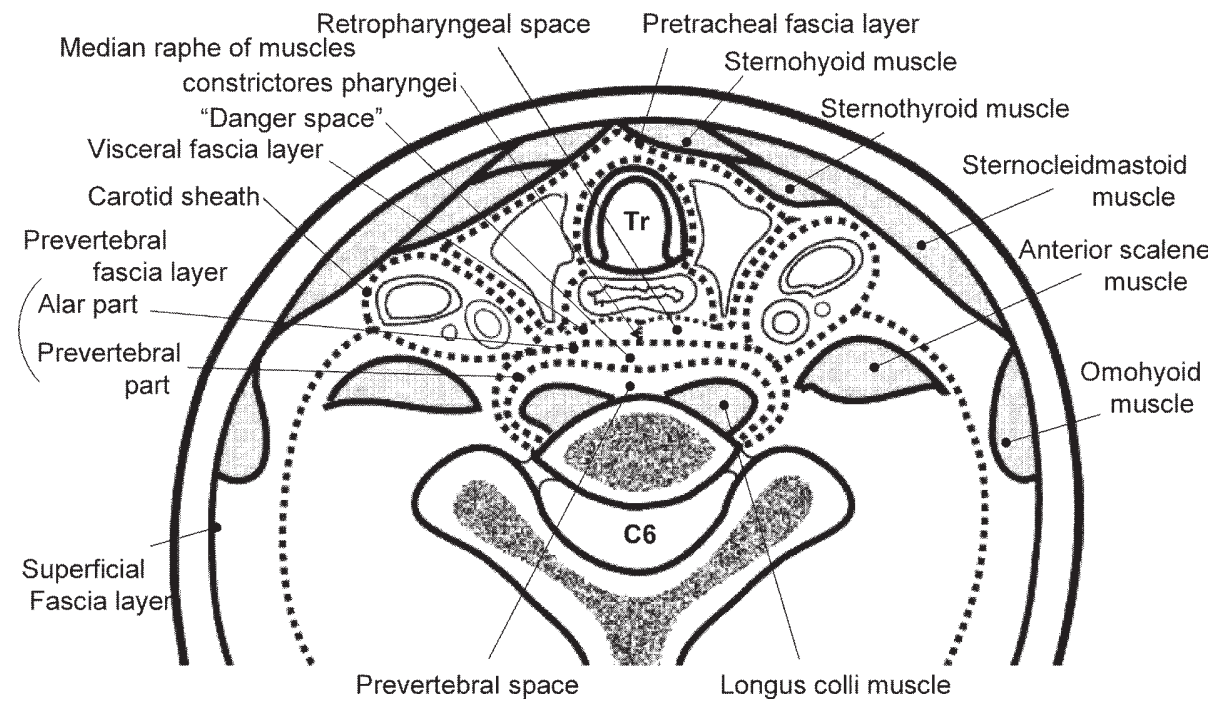

Fig. 1. Schematic diagram of deep neck fascia: The cross-section of the neck at the thyroid. The retropharyngeal space is divided by the alar part of the prevertebralfascia into retropharyngeal and danger spaces. Behind the danger space, the prevertebral fascia covers the paraspinous muscles laterally forming the prevertebral space. Retropharyngeal space is separated by median raphe of muscles constrictores pharyngei into the right and left at the median. Since the retropharyngeal lymph nodes are exterior, the primary retropharyngeal abscess is usually localized in either the left or right (Saitou 1988; Katagiri 2006). 
due to inflammation was also noticed (Figs. 3A and $\mathrm{B}$ ).

On March 30, 2006, the patient was transferred to our hospital ward. The endoscopical examination revealed a clear tumor on the posterior wall of the epipharynx (Fig. 4). Based on the MRI findings, retropharyngeal abscess was diagnosed and arrangements were made for the imme-

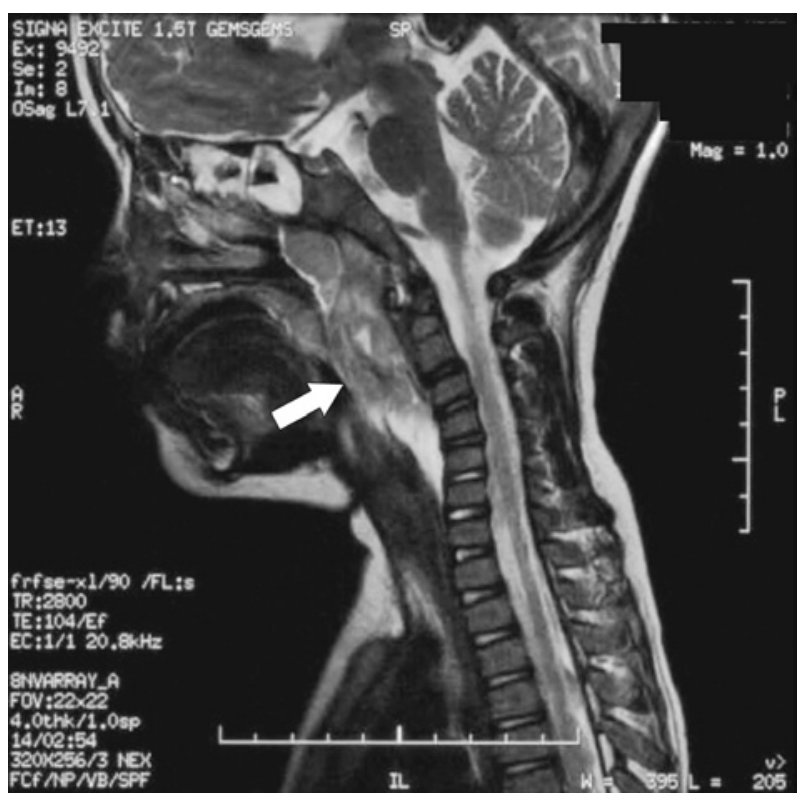

Fig. 2. MRI (vertical). The MRI (T2) vertical findings of the neck shows an apparent tumor mass between the rear of the adenoid and the front of the vestibra body. diate surgical drainage.

\section{Operative findings}

We operated on the patient with the help of a Davis mouth opener, using a method similar to that for adenoidectomy. When the soft palate was turned over, the posterior wall of the epipharynx appeared to be swollen, which was then punctured and drainaged. Culture of the serous fluid yielded Streptococcus mitis, with no noticeable tolerance.

\section{Postoperative course}

After the operation, the patient was given intravenous antibiotics (meropenem trihydrate and clindamycin); her fever as well as inflammatory response on the blood test then improved remarkably. However, the neck pain and torticollis still remained (Fig. 5). An analgesic medication was thus required. On the fourth postsurgical day, the patient was taken to the orthopedic department, where she was given a diagnosis of inflammatory torticollis due to the spread of inflammation to the neck muscles.

Then traction therapy was introduced to decrease immobility due to muscle contracture and adhesion (Fig. 6). On the 8th day after the start of the traction therapy, the symptoms abated. On the 12th day, the patient was transferred to the out-patient care for follow up. On the 25th day, no symptoms of torticollis or neck pain were noticed; the antibiotics were therefore stopped.
A

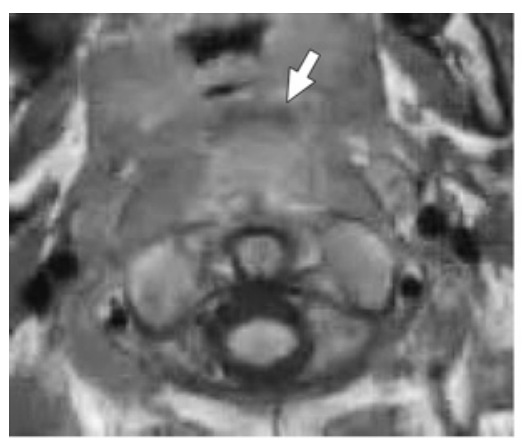

B

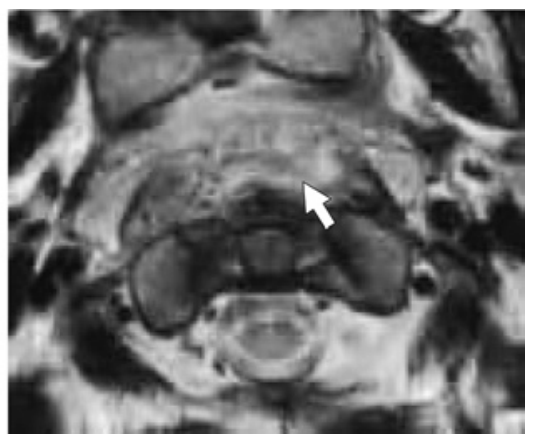

Fig. 3. MRI (horizontal). A: MRI (T1) horizontal findings. Retropharyngeal abscess is seen in the anterior of the prevertebral muscles and the retropharyngeal space (Indicated by the arrow). B: MRI (T2) horizontal findings, prevertebral muscles (musclus longus capitis and musclus longus colli) have conspicuous tumefaction due to inflammation (Indicated by the arrow). 


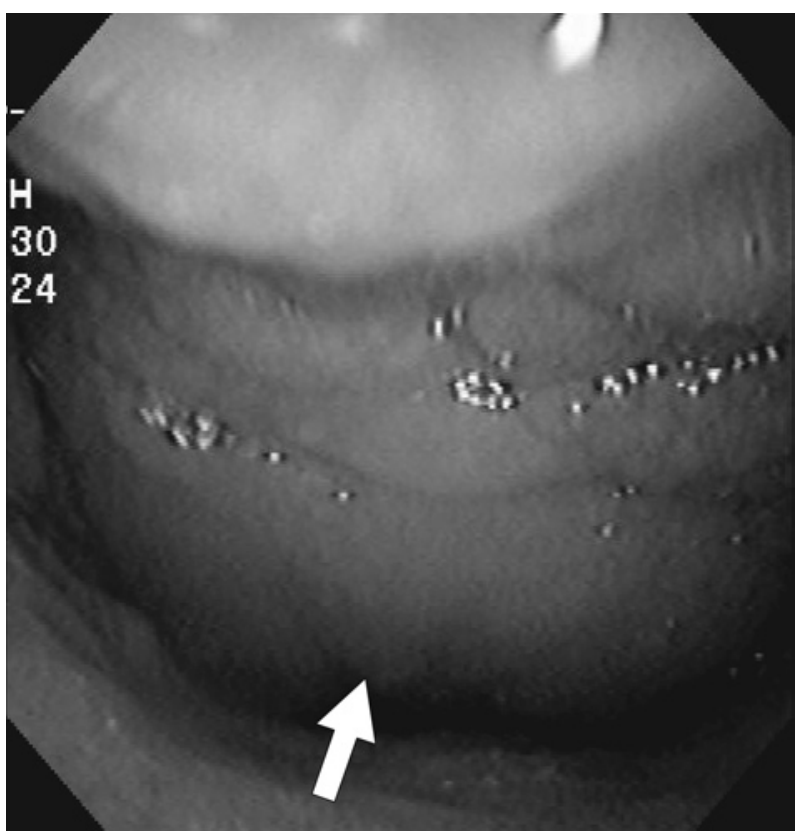

Fig. 4. Findings of Epipharynx: Inspection of the epipharynx through an endoscope at the initial visit. A conspicuous enlargement is shown on the posterior wall of the epipharynx.

The patient got healthier without fear of recurrences of inflammation.

\section{Discussion}

Retropharyngeal abscess commonly develops when, after acute pharyngitis, the retropharyngeal lymph nodes (Gillette's or Henle's lymph node) within the retropharyngeal space become inflamed or tumorigenic. This commonly occurs in childhood (Rouviere 1938; Samuel 1941), partly because the retropharyngeal space is fairly open during childhood and becomes involuted with age, shrinking back after the age of 3 . According to one report, $90 \%$ of such cases have occurred in children below age 6 (Sharma et al. 1998). Meanwhile, it has been reported that though the number of cases of retropharyngeal abscess is decreasing among children due to the advancements of antibiotics, it has been increasing lately among adults. Retropharyngeal abscess in adults has been reported to be due to vertebral osteomyelitis (Barratt et al. 1984), caries of the cervical spine (Bosley 1962), or external injuries caused by endoscopes (Delozier et al. 1986) or

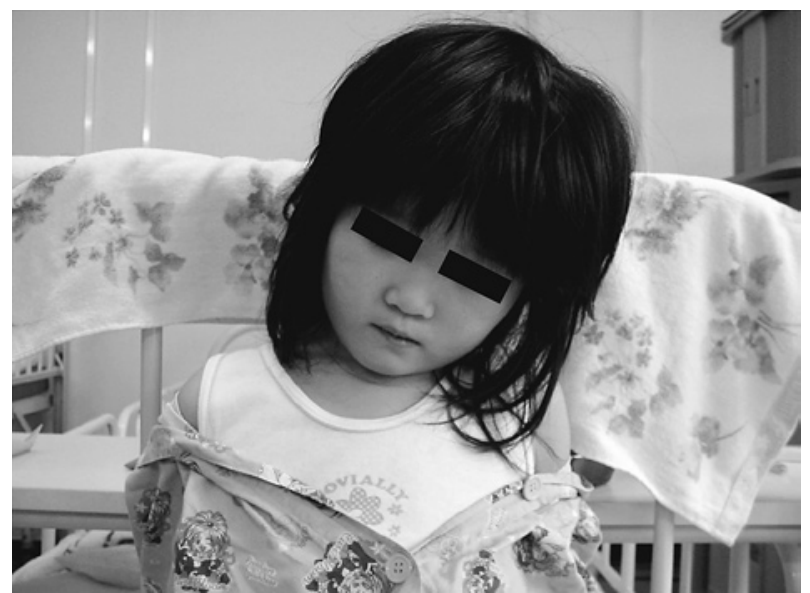

Fig. 5. The Torticollis: The postoperative findings (on the fourth day post-surgery). The torticollis cocking the left part of her head and the complaint of neck pain did not improve.

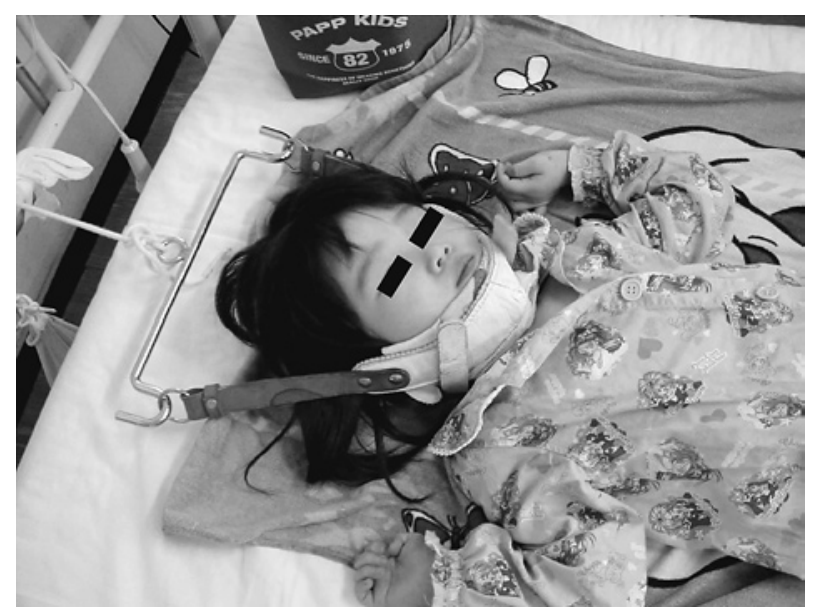

Fig. 6. Traction therapy. Traction therapy (Glisson's traction) was being perfomed at $1 \mathrm{~kg}$, while administering antibiotics. On the 8 th day after the start of the therapy, the symptoms abated and improved.

fish bones (Berger et al. 1990). As for underlying diseases, many patients were diabetics or had been taking steroids for Behçet's syndrome, rheumatism, systemic lupus erythematosus, and so on (Tannebaum 1996).

The retropharyngeal space consists of loose connective tissue between the buccopharyngeal and prevertebral fascia. The retropharyngeal space is divided by the alar prevertebral fascia in 
both front and rear ends into two parts, the anterior and posterior areas (Fig. 1). The front area is called retropharyngeal space, while the rear area is called the "danger space". The alar parts of the prevertebral fascia extend along the cranial base and the cervical vertebrae, bordering the buccopharyngeal mucous membrane at the base. Behind the danger space, on the other side of the spinal prevertebral fascia, is the prevertebral space, which ends at the coccygeal bone (White 1985; Haug et al. 1990; Chow 1992).

Because of this structure, infection of the retropharyngeal lymph nodes does not reach the prevertebral area directly. There are several intervening fascias; so that inflammation of the prevertebral muscles and epidural abscess is believed to occur very rarely even when the abscess remains confined to the retropharyngeal space. When the inflammation is exacerbated and the abscess spreads to the danger space or prevertebral space, a mediastinal abscess or inflammation of the prevertebral muscles in the rear area may ensue (Shumrick and Sheft 1991).

Inflammatory torticollis (stiffness, rigidity, and torsion) is generally caused by swollen lymph nodes due to external injuries or cold (Mezue et al. 2002), but the causes remain unclear in many cases. Mild cases can be cured by a few days' rest; however, when the symptoms fail to improve, neck traction may be needed (Bredenkamp and Maceri 1990). In our search of the literature, we found only a few reports of a retropharyngeal abscess causing inflammatory torticollis (Bredenkamp et al. 1990; Elliott et al. 1992; Harries 1997), they speculated torticollis might be caused by irritation and edema inflammation of the neck muscles. But traction therapy was not perfomed. In this case, as shown in the MRI, there was a wide range of inflammation from the base of the cranium to the level of C6, so the cause of torticollis in this case appeared to be the spreading of inflammation to the musculus longus capitis and musculus longus colli. The horizontal section also suggests the spreading of inflammation to the prevertebral fascia (Fig. 3A and B). Another hypothesis is that the inflammation may have started in the prevertebral muscles, spreading forward into a retropharyngeal abscess. Cases of inflammation of the neck long muscles which started in prevertebral muscles have also been reported (Katagiri et al. 2006). Determining the focus of inflammation from imaging tests is challenging. But in this case, the patient was an infant and the degree of inflammatory reaction was high, so we believe that the retropharyngeal abscess occurred firstly, formed by the inflammation to the prevertebral muscles.

When the neck muscles become severely inflamed, contracture, growth inhibition, or adhesion of muscles may occur, and then torticollis may follow. In our case, we were able to mitigate these symptoms with the help of traction.

Some authorities maintain that as long as the inflammation of retropharyngeal lymph nodes remains at the stage of lymphadenitis without abscess, drainage of pus by incision is not necessary (Glasier et al. 1992; Castillo and Mukherji 1996). In this case, however, for the reasons given above, immediate surgical intervention was thought necessary because the inflammation seemed to have reached the prevertebral fascia at an early stage through the webbed and prespinal fascias. Therefore, if we did not perfom incisional drainage, mediastinitis and epidural abscess would have occurred. Moreover, some recent reports state that the offending bacteria may be anaerobes or beta-lactamase-producers (Brook 1988), so we should perform stab culture and surgical drainage for early diagnoses of the offending bacteria.

The prevertebral fascia borders the cervical spine, which explains the occasional reports of inflammatory torticollis due to the spreading of pyogenic inflammation of the cervical spine (Garcia and Grantham 1960; Ratcliffe 1985; Faidas et al. 1994; Sakamoto et al. 1999). There are also some reports of retropharyngeal abscess causing epidural abscess (Jang and Rhee 1998). As the inflammation becomes severe, it is likely to spread to the cervical spine. In this case, to avoid the infectious spondylitis, a long course of antibiotic therapy was required, as well as careful follow-up from both the otolaryngology and orthopedic departments. Fortunately infectious 
spondylitis was avoided in this case, but there are reports of exacerbation and more serious complications (Hermann et al. 1959; Owens and Bergstrom 1984).

Finally, we should also emphasize a therapy done in cooperation with an orthopedist, including traction therapy.

\section{References}

Barratt, G.E., KooPMann, C.F., Jr. \& Coulthard, S.W. (1984) Retropharyngeal abscess-a ten year experience. Laryngoscope, 94, 455-463.

Berger, S., Elidan, J. \& Gay, I. (1990) Retropharyngeal abscess caused by a traumatic perforation of the hypopharynx by a fishbone. Ann. Otol. Rhinol. Laryngol., 99, 927-928.

Bosley, R.J. (1962) Acute retropharyngeal abscess in children. Report of a case. Laryngoscope, 72, 207-217.

Bredenkamp, J.K. \& Maceri, D.R. (1990) Inflammatory torticollis in children. Arch. Otolaryngol. Head Neck Surg., 116, 310-313.

Brook, I. (1988) Beta-Lactamase-producing bacteria in head and neck infection. Laryngoscope, 98, April, 428-431.

Castillo, M. \& Mukherji, S.K. (1996) Retropharyngeal space infection, infectious cervical adenitis. In: Imaging of the Pediatric Head, Neck, and Spine. Lippincot-Raven, Philadelphia-New York, pp. 594-603.

Chow, A.W. (1992) Life-threatening infections of the head and neck. Clin. Infect. Dis., 14, 991-1004.

Delozier, H.L. \& Sofferman, R.A. (1986) Pyriform sinus fistula: An unusual case of recurrent retropharyngeal abscess and cellulitis. Ann. Otol. Rhinol. Laryngol., 95, 377-382.

Elliott, C.A., Bernard, P.A., Briggs, V.A. \& McDonald, P. (1992) Posterolateral cervicotomy in retropharyngeal abscesses. Laryngoscope, 102, 585-587.

Faidas, A., Ferguson, J.V., Jr., Nelson, J.E. \& Baddour, L.M. (1994) Cervical vertebral osteomyelitis presenting as a retropharyngeal abscess. Clinical Infectious Dis., 18, 992-994.

Garcia, A., Jr. \& Grantham, S.A. (1960) Hematogenous pyogenic vertebral osteomyelitis. J. Bone Joint Surg., 42-A, 429-436.

Glasier, C.M., Stark, J.E., Jacobs, R.F., Mancias, P., Leithiser, R.E., Jr., Seibert, R.W. \& Seibert, J.J. (1992) CT and ultrasound imaging of retropharyngeal abcesses in children.
AJNR AM J. Neuroradiol., 13, 1191-1195.

Harries, P.G. (1997) Retropharyngeal abscess and acute torticollis. J. Laryngol. Otol., 111, 1183-1185.

Haug, R.H., Picard, U. \& Indresano, A.T. (1990) Diagnosis and treatment of the retropharyngeal abscess in adults. $B r . J$. Oral Maxillofac. Surg., 28, 34-38.

Hermann, H.W., Hempel, D.J. \& Erlanson, A.C. (1959) Recurrent retropharyngeal abscess. Lancet, 79, 204-242.

Jang, Y.J. \& Rhee, C.K. (1998) Retropharyngeal abscess associated with vertebral osteomyelitis and spinalepidural abcess. Otol. Head Neck, 119, 705-708.

Katagiri, K., Ishidoya, M. \& Mastutani, S. (2006) A case of calcific retropharyngeal tendinitis which required a differential diagnosis of retropharyngeal abscess. Journal of Department of Japanese Red Cross Sendai Hospital, 15, 87-91. (in Japanese)

Mezue, W.C., Taha, Z.M. \& Bashir, E.M. (2002) Fever and acquired torticollis in hospitalized children. J. Laryngol. Otol., 116, 280-284.

Owens, O.T. \& Bergstrom, L. (1984) Recurrent Retropharyngeal abscess. Arch. Otolaryngol., 110, 337-338.

Ratcliffe, J.F. (1985) Anatomic bases for the pathogenesis and radiologic features of vertebral osteomyelitis and its differentiation from childfood discitis. Acta Radiol. (Diagno.), 26, 137-143.

Rouviere, H. (1938) Anatomy of the human lymphatic system.1st ed., Ann Arbor, MI, Edwards Borthers, 1-82.

Saitou, H. (1988) Retorpharyngeal abscess. JOHNS, 4, 62-65. (in Japanese)

Sakamoto, M., Ichimura, K., Tayama, N., Nakamura, M. \& Inokuchi, K. (1999) Cervical vertebral osteomyelitis revisited: A case of retropharyngeal abscess and progressive muscle weakness. Otol. Head Neck Surg., 121, 657-660.

Samuel, I. (1941) Anatomic-phathologic studies of retropharyngeal (peripharyngeal) abscess, Arch. Otolaryngol. Head Neck, 33, 31-34.

Sharma, H.S., Kurl, D.N. \& Hamzah, M. (1998) Retropharyngeal abscesses; recent trends. Auris Nasus Larynx, 25, 403-406.

Shumrick, K.A. \& Sheft, S.A. (1991) Deep neck infections. In: Otolaryngology, Head and Neck, 3rd ed., 3, W.B. Saunders, Philadelphia, pp. 2545-2563.

Tannebaum, R.D. (1996) Adult Retropharyngeal abscess: A case report and reveiw of the literatrure. J. Emerg. Med., 14, 147-158.

White, B. (1985) Deep neck infections and respiratory distress in children. Ear Nose Throat J., 64, 30-38. 